MODELING, IDENTIFICATION AND CONTROL, 1995, vOL. 16, NO. 4, 213-232

doi:10.4173/mic.1995.4.3

\title{
Semi-empirical modeling of non-linear dynamic systems through identification of operating regimes and local models
}

\author{
TOR A. JOHANSEN†‡ and BJARNE A. FOSS $\dagger$ \\ Keywords: Non-linear systems, system identification, fuzzy modeling, heuristic
search algorithms
}

An off-line algorithm for semi-empirical modeling of non-linear dynamic systems is presented. The model representation is based on the interpolation of a number of simple local models, where the validity of each local model is restricted to an operating regime, but where the local models yield a complete global model when interpolated. The input to the algorithm is a sequence of empirical data and a set of candidate local model structures. The algorithm searches for an optimal decomposition into operating regimes, and local model structures. The method is illustrated using simulated and real data. The transparency of the resulting model and the flexibility with respect to incorporation of prior knowledge is discussed.

\section{Introduction}

The problem of identifying a mathematical model of an unknown system from a sequence of empirical data is a fundamental one which arises in many branches of science and engineering. The complexity of solving such a problem depends on many factors, such as a priori knowledge, quality and completeness of the data sequence, and required model form and accuracy.

A rich non-linear model representation based on patching together a number of simple local models into a complex global model is suggested in Takagi and Sugeno (1985), Stokbro et al. (1990), Jones et al. (1991), Jacobs et al. (1991), Johansen and Foss (1993a). With this representation, the modeling problem is basically to decompose the operating range of the system into a set of operating regimes, the identification of simple local models within each regime, and the interpolation of the local models to get a global model. This is an example of the classical divide-and-conquer strategy, where a complex problem is decomposed into simple subproblems that can be solved independently, and whose solutions add up to solve the complex problem. In Johansen and Foss $(1993 a, b)$ we have focused on the use of system knowledge for regime decomposition. The aim of the present paper is to report on a algorithm that automatically finds a decomposition and local models based on empirical data.

The paper is organized as follows. The problem is formulated in section 2, before an empirical modeling algorithm is developed in section 3 . The algorithm is applied to simulated and real data in section 4 , and in section 5 the role of prior knowledge and the transparency of identified models are discussed. A comparison to related work follows, along with some concluding remarks.

Received 1 July 1995.

† Department of Engineering Cybernetics, Norwegian Institute of Technology, 7034 Trondheim, Norway.

† Present address: SINTEF Automatic Control, N-7034 Trondheim, Norway.

In K. Hunt, G. Irwin and K. Warwick (Eds.) Neural Network Engineering in Dynamic Control Systems, Springer Verlag, London 1995. Reprinted with kind permission from Springer-Verlag. 


\section{Problem formulation}

We address the problem of identifying a model of an unknown non-linear system on the basis of a sequence of $l$ input/output-pairs

$$
\mathscr{D}_{l}=((u(1), y(1)),(u(2), y(2)), \ldots,(u(l), y(l)))
$$

where $u(t) \in R^{r}$ and $y(t) \in R^{m}$ are the input and output vectors of the system, respectively. We demote by $\mathscr{D}_{t}$ the subsequence of $\mathscr{D}_{l}$ containing data up to and including time $t \leqslant l$. First, consider static models

$$
y(t)=f(u(t))+e(t)
$$

where $e(t) \in R^{m}$ is zero-mean noise, and $f$ is an unknown function to be estimated. An approximation $\hat{f}$ to $f$ suggests the predictor $\hat{y}(t \mid u(t))=\hat{f}(u(t))$ which gives the prediction error

$$
\varepsilon(t)=y(t)-\hat{y}(t \mid u(t))=(f(u(t))-\hat{f}(u(t)))+e(t) .
$$

Next, consider a stable dynamic system represented by the NARMAX (non-linear ARMAX) model

$$
\begin{gathered}
y(t)=f\left(y(t-1), \ldots, y\left(t-n_{y}\right), u(t-1), \ldots, u\left(t-n_{u}\right),\right. \\
\left.e(t-1), \ldots, e\left(t-n_{e}\right)\right)+e(t)
\end{gathered}
$$

where $e(t) \in R^{m}$ is zero-mean noise, and $n_{y}, n_{u}$, and $n_{e}$ are non-negative integers. Given an approximation $\hat{f}$ to the function $f$, a one-step-ahead predictor $\hat{y}\left(t \mid \mathscr{D}_{t-1}\right)$ can be formulated. The predictor and prediction error are defined by

$$
\begin{aligned}
\hat{y}\left(t \mid \mathscr{D}_{t-1}\right) & =\hat{f}\left(y(t-1), \ldots, y\left(t-n_{y}\right), u(t-1), \ldots, u\left(t-n_{u}\right), \varepsilon(t-1), \ldots, \varepsilon\left(t-n_{e}\right)\right) \\
\varepsilon(t) & =y(t)-\hat{y}\left(t \mid \mathscr{D}_{t-1}\right)
\end{aligned}
$$

The motivation behind this predictor is that while the noise sequence $e$ is unknown, $\varepsilon(t) \rightarrow e(t)$ as $t \rightarrow \infty$, if $\hat{f}=f$ and the model is invertible.

Finally, we consider state-space models

$$
\begin{gathered}
x(t+1)=g(x(t), u(t))+v(t) \\
y(t)=h(x(t))+w(t)
\end{gathered}
$$

where $x(t)$ is a state-vector, and $v(t)$ and $w(t)$ are zero-mean disturbance and noise vectors of appropriate dimensions. In this case, the model is defined by the functions $g$ and $h$. Again, using approximations $\hat{g}$ and $\hat{h}$, it is possible to construct a one-step-ahead predictor $\hat{y}\left(t \mid \mathscr{D}_{t-1}\right)$ using the extended Kalman-filter approach, e.g. Ljung (1987)

$$
\begin{aligned}
\hat{x}\left(t \mid \mathscr{D}_{t-1}\right) & =\hat{g}\left(\hat{x}\left(t-1 \mid \mathscr{D}_{t-2}\right), u(t-1)\right)+K(t-1) \varepsilon(t-1) \\
\hat{y}\left(t \mid \mathscr{D}_{t-1}\right) & =\hat{h}\left(\hat{x}\left(t \mid \mathscr{D}_{t-1}\right)\right) \\
\varepsilon(t) & =y(t)-\hat{y}\left(t \mid \mathscr{D}_{t-1}\right)
\end{aligned}
$$

where $K(t)$ is the Kalman-filter gain matrix. This matrix will depend explicitly on the time, the functions $\hat{g}$ and $\hat{h}$, and the covariance matrices of the disturbance and noise sequences. 


\subsection{A generalized framework}

In all these cases, we can write the model on the form

$$
\eta(t)=f(\xi(t))+e(t)
$$

where $\eta(t) \in R^{\bar{m}}$ is a generalized output-vector, $\xi(t) \in R^{\tilde{r}}$ is a generalized input-vector, and $e(t) \in R^{\tilde{m}}$ is zero-mean noise. We denote the space $R^{r}$ the input space. In the static model case (1), the input and output vectors equal the generalized input and output vectors. In the NARMAX case (2), the generalized input vector contains delayed input and output vectors in addition to delayed noise vectors, while the generalized output equals the system output. If noise terms $e(t-1), \ldots, e\left(t-n_{e}\right)$ are present, the generalized input vector is partially unknown and cannot be found exactly from the data $\mathscr{D}_{1}$. For state-space models (3)-(4), neither the generalized input nor the generalized output vectors can be found exactly, because they contain the unknown state vector. The purpose of the formulation (5) with the generalized input and output vectors is to write the model in a generic form with one unknown function $f$. The problem we address is to estimate this function, and since the function immediately gives the model equations, this also solves the system identification problem. Notice that the fact that the generalized inputs and outputs may not be exactly known does not complicate this problem too much, since the model parameters can still be estimated from the input/output data using a prediction error approach with the predictors described above (Ljung 1987).

\subsection{Model representation}

In Takagi and Sugeno (1985), Stokbro et al. (1990), Jones et al. (1991), Jacobs et al (1991), Johansen and Foss (1993a, b) a non-linear model representation with good interpolation and extrapolation properties is described. It is based on the decomposition of the system's operating range into a number of smaller operating regimes, and the use of simple local models to describe the system within each regime. A global model is formed by interpolating the local models using smooth interpolation functions, that depend on the operating point.

We define the system's operating point at time $t$ as $z(t)=\left(z_{1}(t), \ldots, z_{d}(t)\right)^{\mathrm{T}} \in Z=R^{d}$, where typically $d \leqslant \bar{r}$ and the operating space $Z$ is a subspace or sub-manifold of the input space. It is assumed the $\xi$ and $z$ are related by a known bounded mapping $H$ so that $z=H(\xi)$. Typically, $Z$ and $H$ are designed such that the operating point $z(t)$ characterizes different modes of behaviour of the system under different operating
conditions. The design of $Z$ and $H$ is discussed in more detail in section 4 , and in Johansen (1994). Suppose $Z$ is decomposed into $N$ disjoint sets $\left\{Z_{i}\right\}_{i \in I_{N}}$ (regimes) so
that

$$
Z=\bigcup_{i \in I_{N}} Z_{i}
$$

for some index set $I_{N}=\left\{i_{1}, \ldots, i_{N}\right\}$ with $N$ elements. Assume that for each regime $Z_{i}$ we have a local model structure defined by the function $\hat{f}_{i}\left(\xi ; \theta_{i}\right.$ ) (parameterized by the vector $\left.\theta_{i}\right)$ and a local model validity function $\rho_{i}(z) \geqslant 0$ which indicates the relative validity of the local model as a function of $z$. In addition to being smooth, $\rho_{i}$ is designed to have the property that $\rho_{i}(z)$ is close to zero if $z \notin Z_{i}$. Furthermore, it is assumed that for all $z \in Z$ there exists an $i \in I_{N}$ so that $\rho_{i}(z)>0$, to ensure completeness of the model. 
A global model can be formed as

$$
\begin{aligned}
& \hat{f}(\xi)=\sum_{i \in I_{N}} \hat{f}_{i}\left(\xi ; \theta_{i}\right) w_{i}(z) \\
& w_{i}(z)=\rho_{i}(z) / \sum_{j \in I_{N}} \rho_{j}(z)
\end{aligned}
$$

where the functions $\left\{w_{i}\right\}_{i \in l_{N}}$ are called interpolation functions. This representation is discussed in detail in Johansen and Foss (1993a), and it is shown that if the model validity functions and operating space are adequately chosen, then any continuous function $f$ can be uniformly approximated to an arbitrary accuracy on any compact subset of the input space using this representation. A model structure based on a decomposition into $N$ regimes is written

$$
\mathscr{M}_{N}=\left\{\left(Z_{i}, \rho_{i}, \hat{f}_{i}\right)\right\}_{i \in I_{N}}
$$

This is somewhat redundant, since there is a close (but not necessarily one-to-one) relationship between $Z_{i}$ and $\rho_{i}$. With this representation, the modeling problem consists of the following subproblems:

1. Choose the variables with which to characterize the operating regimes, i.e. the operating space $Z$ and mapping $H$.

2. Decompose $Z$ into regimes, and choose local model structures.

3. Identify the local model parameters for all regimes.

In Johansen and Foss (1993a, b, and Johansen 1994) it is demonstrated that in some cases, some coarse qualitative system understanding is sufficient to carry out this procedure. In the following sections we propose an algorithm that requires significantly less prior knowledge in order to automatically decompose $Z$, choose local model structures, and construct interpolation functions.

\subsection{Model structure identification criteria}

Let a model structure $\mathscr{M}$ of the form (8) be given. Notice that in a model structure, the model parameters $\theta^{\mathrm{T}}=\left(\theta_{i_{1}}^{\mathrm{T}}, \ldots, \theta_{i_{N}}^{\mathrm{T}}\right)$ are considered unknown. The model structure $\mathscr{M}$ together with the admissible parameter set $\Theta_{\mathscr{M}}$ generate a model set $\{\mathscr{M}(\theta)$; $\left.\theta \in \Theta_{\mathscr{M}}\right\}$. In this section, we will discuss how different model structures can be compared using a sequence of empirical data to estimate their expected prediction performance. Let an unknown future data sequence be denoted $\mathscr{D}_{t}^{*}$, and assume $\mathscr{D}_{t}^{*}$ and $\mathscr{D}_{l}$ are uncorrelated. We introduce the notation

$$
\begin{gathered}
y(t)=y^{*}\left(\mathscr{D}_{t-1}^{*}\right)+e(t) \\
\varepsilon(t \mid \mathscr{M}, \theta)=y(t)-\hat{y}\left(t \mid \mathscr{D}_{t-1}^{*}, \mathscr{M}, \theta\right)
\end{gathered}
$$

where $y^{*}\left(\mathscr{D}_{t-1}^{*}\right)$ is the deterministic (predictable) component of the system output, $e(t)$ is the stochastic (unpredictable) component, and $\varepsilon(t \mid S, \theta)$ is a prediction error. Let $\hat{\theta}_{\mathscr{A}}$ be the parameter estimate that minimizes the prediction error criterion

$$
J_{\mathscr{M}}(\theta)=\frac{1}{l} \sum_{t=1}^{l} \operatorname{trace}\left(\varepsilon(t \mid \mathscr{M}, \theta) \varepsilon^{\mathrm{T}}(t \mid \mathscr{M}, \theta)\right)
$$

and let $E_{\mathscr{D}}$ and $E_{\mathscr{D}^{*}}$ denote expectations with respect to $\mathscr{D}_{l}$ and $\mathscr{D}_{t}^{*}$, respectively. The future prediction error is given by

$$
\varepsilon^{*}\left(t \mid \mathscr{M}, \hat{\theta}_{\mathscr{M}}\left(\mathscr{D}_{l}\right)\right)=y^{*}\left(\mathscr{D}_{t-1}^{*}\right)-\hat{y}\left(t \mid \mathscr{D}_{t-1}^{*}, \mathscr{M}, \hat{\theta}_{-\mu}\left(\mathscr{D}_{1}\right)\right)+e(t)
$$


where the dependence of $\mathscr{D}_{\mathbb{l}}$ on $\hat{\theta}_{\mathscr{M}}$ has been written explicitly. The expected squared prediction error is defined by

$$
\Sigma(\mathscr{M})=E_{\mathscr{D}} E_{\mathscr{D}}\left(\varepsilon^{*}\left(t \mid \mathscr{M}, \hat{\theta}_{\mathscr{M}}(\mathscr{D} t)\right)\right)\left(\varepsilon^{*}\left(t \mid \mathscr{M}, \hat{\theta}_{\mathscr{M}}(\mathscr{D} l)\right)\right)^{\mathrm{T}} .
$$

Assuming $e(t)$ is white noise that is uncorrelated with $\mathscr{D}_{t-1}^{*}$ and $\mathscr{D}_{l}$, we get the following bias/variance decomposition of this expected squared prediction error

$$
\begin{aligned}
& \Sigma(\mathscr{M})=E_{\mathscr{P}^{*}}\left(y^{*}\left(\mathscr{D}_{t-1}^{*}\right)-E_{\mathscr{Q}} \hat{y}\left(t \mid \mathscr{D}_{t-1}^{*}, \mathscr{M}, \hat{\theta}_{\mathscr{M}}\left(\mathscr{D}_{l}\right)\right)\right) \\
& \times\left(y^{*}\left(\mathscr{D}_{t-1}^{*}\right)-E_{\mathscr{Q}} \hat{y}\left(t \mid \mathscr{D}_{t-1}^{*}, \mathscr{M}, \hat{\theta}_{\mathscr{M}}\left(\mathscr{D}_{l}\right)\right)\right)^{\mathrm{T}} \\
& +E_{\mathscr{D}^{*}} E_{\mathscr{C}}\left(\hat{y}\left(t \mid \mathscr{D}_{t-1}^{*}, \mathscr{M}, \hat{\theta}_{\mathscr{M}}\left(\mathscr{D}_{l}\right)\right)-E_{\mathscr{D}} \hat{y}\left(t \mid \mathscr{D}_{t-1}^{*}, \mathscr{M}, \hat{\theta}_{\mathscr{M}}\left(\mathscr{D}_{l}\right)\right)\right) \\
& \times\left(\hat{y}\left(t \mid \mathscr{D}_{t-1}^{*}, \mathscr{M}, \hat{\theta}_{\mathscr{M}}\left(\mathscr{D}_{l}\right)\right)-E_{\mathscr{Q}} \hat{y}\left(t \mid \mathscr{D}_{t-1}^{*}, \mathscr{M}, \hat{\theta}_{\mathscr{M}}\left(\mathscr{D}_{l}\right)\right)\right)^{\mathrm{T}} \\
& +E_{\mathscr{Q} *}\left(e(t) e^{\mathrm{T}}(t)\right)
\end{aligned}
$$

The first term is the squared bias caused by a too simple model structure. The second term is the variance that is present because the best model in the model set $\{\mathscr{M}(\theta)$; $\left.\theta \in \Theta_{\mathscr{M}}\right\}$ cannot in general be identified on the basis of the finite data sequence $\mathscr{D}_{\mathbb{l}}$. Finally, the third term is the unpredictable component of the system output. Notice that the first term does not depend on the data $\mathscr{D}_{l}$, while the third term depends neither on the data $\mathscr{D}_{l}$, nor on the model structure $\mathscr{M}$. It is evident that a small bias requires a complex model structure, in general. On the other hand, a small variance requires a simple model structure, with few parameters compared to the number of observations $l$. The perfect model is characterized both by small bias and variance, and this appears to be conflicting goals for a small $l$. This is known as the bias/variance dilemma, see Fig. 1.

The model set will be based on a set of functions that can approximate any smooth function uniformly on a compact subset of the input space. This is obviously a desirable property of the model set, but also a cause for some problems. The richness implies that there will exist models in the model set that may make the bias small. However, the finite amount of data will give large variance for such models, and such a model will be fitted to represent not only the system, but also the particular realization of the noise.



Figure 1. Typical relationship between bias, variance and model structure complexity, when it is assumed that model structure complexity can be measured by a real number. Of course, this illustration is simplified, since two different models of exactly the same complexity
may have different bias and variance 
In other words, the model may give very good prediction of $\mathscr{D}_{1}$, but poor prediction capability when applied to $\mathscr{D}_{t}^{*}$. This is known as over-fitting, and is caused by too many degrees of freedom in the model structure. It is therefore important that a model structure with an appropriate number of degrees of freedom is found, in the sense that it balances bias and variance. We will base the model structure identification algorithm on statistical criteria that have this property.

The mean square error (MSE) criterion is defined by

$$
J_{\text {MSE }}(\mathscr{M})=\operatorname{trace}(\Sigma(\mathscr{M}))
$$

Minimizing $J_{M S E}$ will lead to a parsimonious model structure, but with a finite sequence of data $\mathscr{D}_{l}$, the problem is ill-posed. The reason is simply that $J_{M S E}$ cannot be computed since the probability distribution for the prediction error is unknown. An alternative would be to minimize the average squared residuals (ASR) criterion with respect to the model structure

$$
J_{\mathrm{ASR}}(\mathscr{M})=\operatorname{trace}\left(\frac{1}{l} \sum_{t=1}^{l} \varepsilon\left(t \mid \mathscr{M}, \hat{\theta}_{\mathscr{M}}\right) \varepsilon\left(t \mid \mathscr{M}, \hat{\theta}_{\mathscr{M}}\right)^{\mathrm{T}}\right)
$$

For finite $l, J_{A S R}$ may be a strongly biased estimate of $J_{M S E}$, since the prediction performance is measured using the same data as those to which the parameters are fitted, and the law of large numbers is not valid because this introduces a strong dependence. Hence, the use of $J_{A S R}$ for structure identification will not, in general, lead to a parsimonious model. We will in the following present several criteria that are far better estimates of $J_{M S E}$ than $J_{A S R}$.

If a separate data sequence $\mathscr{D}_{l}^{*}$ (independent of $\mathscr{D}_{l}$ ) is known, an unbiased estimate of $J_{M S E}$ can be found by computing the empirical average squared prediction error that results when the model fitted to the data $\mathscr{D}_{l}$ is used to predict the data $\mathscr{D}_{l}^{*}$. This is the simplest and perhaps most reliable procedure, but suffers from the drawback that a significantly larger amount of data is required. Experiments and collection of data are major costs for many modeling problems. We therefore proceed with some alternatives that allow the data $\mathscr{D}_{1}$ to be recycled in order to find good estimates of $J_{M S E}$. First we consider the final prediction error criterion (FPE) (Akaike 1969), given by

$$
J_{\text {FPE }}(\mathscr{M})=\frac{1+p(\mathscr{M}) / l}{1-p(\mathscr{M}) / l} J_{A S R}(\mathscr{M})
$$

where $p(\mathscr{M})$ is the effective number of parameters (degrees of freedom) in the model structure. $J_{F P E}$ is an estimate of $J_{M S E}$, and penalizes model complexity relative to the length of the available data sequence through the term $p(\mathscr{M}) / l$. A major restriction is that the predictor is assumed to be linearly parameterized. A non-linear generalization is given in Larsen (1992). An alternative criterion can be formulated using cross-validation (Stone 1974, Stoica et al. 1986). The idea is to fit the parameters to different subsets of the data set. and test the prediction performance of the model structure on the remaining (presumed independent) data. Cross-validation may give a reasonable approximation to the use of independent data for selecting the model structure, at the cost of extra computations. The computational complexity can be considerably reduced if the predictor is a linear function of its parameters, or in general by using one of the approximate cross-validation criteria in Stoica et al. (1986). It is shown that the approximate criteria are asymptotically equivalent to FPE, as $l \rightarrow \infty$. Another approximation to cross-validation is the Generalized Cross Validation (GCV) criterion (Craven and Wahba 1979) 


$$
J_{\mathrm{GCV}}(\mathscr{M})=\frac{1}{(1-p(\mathscr{M}) / l)^{2}} J_{A S R}(\mathscr{M})
$$

which is easily seen to be asymptotically equivalent to FPE, and also assumes linear parameterization of the predictor. Any one of these criteria can be applied with the structure identification algorithm we will present in the next section.

\section{System identification}

Let a set of candidate local model structures $\mathscr{L}=\left\{L_{1}, L_{2}, \ldots, L_{N_{L}}\right\}$ be given. $L_{i}$ is a parameterized function that defines a local model structure, cf. (8).

\subsection{The set of model structures candidates}

Assume the input- and output-samples in $\mathscr{D}_{l}$ are bounded. Then the system's operating range $Z$ can be approximated by the $d$-dimensional box

$$
Z_{1}=\left[z_{1,1}^{\min }, z_{1,1}^{\max }\right] \times \ldots \times\left[z_{1, d}^{\min }, z_{1, d}^{\max }\right]
$$

where $z(t) \in Z_{1}$ for all $t \in\{1, \ldots, l\}$, since $H$ is a bounded mapping. Notice that the resulting model will extrapolate and can be applied for operating points outside $Z_{1}$. Next, we consider the problem of decomposing $Z_{1}$ into regimes.

Consider the possible decompositions of the set $Z_{1}$ into two disjoint subsets $Z_{11}$ and $Z_{12}$ with the property $Z_{1}=Z_{11} \cup Z_{12}$. We restrict the possibilities by the constraint that the splitting boundary is a hyper-plane orthogonal to one of the natural basis-vectors of $R^{d}$, i.e.

$$
\begin{aligned}
& Z_{11}=\left\{z \in Z_{1} \mid z_{d_{1}}<\zeta_{1}\right\} \\
& Z_{12}=\left\{z \in Z_{1} \mid z_{d_{1}} \geqslant \zeta_{1}\right\}
\end{aligned}
$$

for some dimension index $d_{1} \in\{1, \ldots, d\}$ and splitting point $\zeta_{1} \in\left[z_{1, d_{1}}^{\min }, z_{1, d_{1}}^{\max }\right]$. Local model validity functions for the two regimes are defined by the recursion

$$
\begin{aligned}
& \rho_{11}(z)=\rho_{1}(z) b\left(z_{d_{1}}-\bar{z}_{11, d_{1}} ; \lambda_{11}\right) \\
& \rho_{12}(z)=\rho_{1}(z) b\left(z_{d_{1}}-\bar{z}_{12, d_{1}} ; \lambda_{12}\right)
\end{aligned}
$$

where $\bar{z}_{i, d_{1}}=0 \cdot 5\left(z_{i, d_{1}}^{\min }+z_{i, d_{1}}^{\max }\right)$ for $i \in\{11,12\}$ is the centre point of $Z_{i}$ in the $d_{1}$-direction. The function $b(r, \lambda)$ is a scalar basis-function with scaling parameter $\lambda$, and the local model validity function associated with the regime $Z_{1}$ is $\rho_{1}(z)=1$. The scaling parameters are chosen by considering the overlap between the local model validity functions. For $i \in\{11,12\}$, we choose $\lambda_{i}=0.5 \gamma\left(z_{i, d_{1}}^{\max }-z_{i, d_{1}}^{\min }\right)$ where $\gamma$ is a design parameter that typically takes on a value between 0.25 and $2 \cdot 0$. There will be almost no overlap when $\gamma=0.25$, and large overlap when $\gamma=2 \cdot 0$. For each dimension index $d_{1} \in\{1, \ldots, d\}$ we represent the interval $\left[z_{1, d_{1}}^{\min }, z_{1, d_{1}}^{\max }\right]$ by a finite number of $N_{1}$ points uniformly covering the interval. Now $d_{1}, \zeta_{1}, L_{1, v}$, and $L_{2, w}$ define a new model structure, where the regime $Z_{1}$ is decomposed according to the dimension index $d_{1}$ at the point $\zeta_{1}$, and the two local model structures are $L_{1, v}$ and $L_{2, w}$. Formally, the set of candidate model structures $\mathscr{S}_{n}$ with regimes is given by

$$
\begin{aligned}
& \mathscr{S}_{1}=\left\{\left\{\left(Z_{1}, \rho_{1}, L_{j}\right)\right\} ; j \in\left\{1,2, \ldots, N_{L}\right\}\right\} \\
& \mathscr{S}_{2}=\left\{\left\{\left(Z_{11}^{i}, \rho_{11}^{i}, L_{j}\right),\left(Z_{12}^{i}, \rho_{12}^{i}, L_{k}\right)\right\} ; i \in\left\{1,2, \ldots, d N_{1}\right\}, j, k \in\left\{1,2, \ldots, N_{L}\right\}\right\}
\end{aligned}
$$




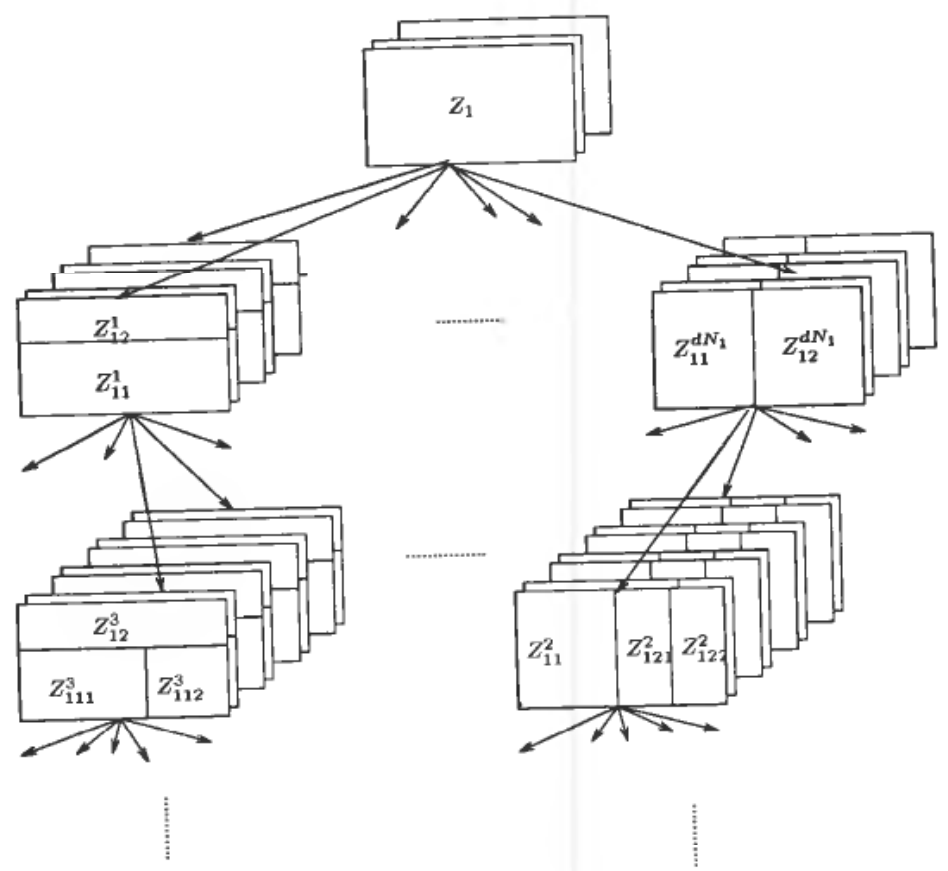

Figure 2. Model structure search tree illustrating possible decompositions into regimes and choices of local model structures. Each level in the tree corresponds to the possible decompositions into one more regime than at the previous level, i.e. the model structure sub-sets $\mathscr{S}_{1}, \mathscr{S}_{2}, \mathscr{S}_{3}, \ldots$ The sub-set of model structures at each 'super-node' in the tree corresponds to a fixed decomposition into regimes, but to different combinations of local model structures. The suggested algorithm will search this tree starting at the top (corresponding to one local model covering the whole operating space), and selecting a decomposition at each level through a sequence of 'locally exhaustive' searches of depth $n *$.

$$
\begin{gathered}
\mathscr{S}_{3}=\left\{\left\{\left(Z_{11}^{i}, \rho_{11}^{i}, L_{j}\right),\left(Z_{121}^{m}, \rho_{121}^{m}, L_{k}\right),\left(Z_{122}^{m}, \rho_{122}^{m}, L_{n}\right)\right\}\right. \\
\left.\quad i, m \in\left\{1,2, \ldots, d N_{1}\right\}, j, k, n \in\left\{1,2, \ldots, N_{L}\right\}\right\} \\
\cup\left\{\left\{\left(Z_{111}^{m}, \rho_{111}^{m}, L_{k}\right),\left(Z_{112}^{m}, \rho_{112}^{m}, L_{n}\right),\left(Z_{12}^{i}, \rho_{12}^{i}, L_{j}\right)\right\} ;\right. \\
\left.\quad i, m \in\left\{1,2, \ldots, d N_{1}\right\}, j, k, n \in\left\{1,2, \ldots, N_{L}\right\}\right\} \\
\mathscr{S}_{4}=\ldots
\end{gathered}
$$

The model structure set is now $\mathscr{S}=\mathscr{S}_{1} \cup \mathscr{P}_{2} \cup \mathscr{P}_{3} \cup \ldots$. The model structure set is illustrated as a search tree in Fig. 2. Strictly speaking, the model structure set is not a tree, since different sequences of decompositions sometimes lead to the same model structure. However, we choose to represent it as a tree, for the sake of simplicity. Now the structure identification problem can be looked upon as a multi-step decomposition process, where at each step one regime from the previous step is decomposed into two sub-regimes. Such an approach will lead to a sequence of model structures $\mathscr{M}_{1}, \mathscr{M}_{2}, \ldots, \mathscr{M}_{n}$ where the model structure $\mathscr{U}_{i+1}$ has more degrees of freedom than $\mathscr{M}_{i}$. Due to the normalization of the local model validity functions, the model set is usually not strictly hierarchical, in the sense that $\mathscr{U}_{i}$ cannot be exactly represented using $\boldsymbol{M}_{i+1}$. However, the increasing degrees of freedom define an approximately hierarchical structure. 


\subsection{Basic search algorithm}

The problem is now to search through the set $\mathscr{S}$ for the best possible model structure. The estimate of the parameters in the model structure $\mathscr{M}$ is defined by a prediction error
criterion

$$
\hat{\theta}=\arg \min _{\theta} J_{\mathscr{M}}(\theta)
$$

where it has been assumed that the minimum exists. This can be ensured by restricting the parameters to a compact set. Now, the chosen structure identification criterion is
written $J^{\prime}(\mathscr{M})$. We define for a given $n \geqslant 1$

$$
\mathscr{M}_{n}=\arg \min _{\mathscr{M} \in \mathscr{S}_{n}} J^{\prime}(\mathscr{M})
$$
Consider the following extended horizon search algorithm, where the integer $n^{*} \geqslant 1$ is
called the search horizon.

\subsubsection{Search algorithm}

1. Start with the regime $Z_{1}$. Let $n=1$.

2. At each step $n \geqslant 1$, find a sequence of decompositions $\mathscr{M}_{n}, \mathscr{M}_{n+1}, \ldots, \mathscr{M}_{n+n^{*}}$ that solves the optimization problem

$$
\min _{\mathscr{M} \in \mathscr{S}_{n+n^{*}}} J^{\prime}(\mathscr{M})
$$
3. Restrict the search tree by keeping the decomposition that leads to $\mathscr{M}_{n+1}$
fixed for the future.

4. If

$$
J^{\prime}\left(\mathscr{M}_{n}\right)>\min _{k \in\left\{1,2, \ldots, n^{*}\right\}} J^{\prime}\left(\mathscr{M}_{n+k}\right)
$$

then increment $n$ and go to 2 . Otherwise, the model structure $\mathscr{M}_{n}$ is chosen.

Referring to Fig. 2, this algorithm will search the tree starting at the top (corresponding to one local model covering the whole operating space), and selecting a decomposition at each level through a sequence of 'locally exhaustive' searches of depth $n^{*}$. If $n^{*}=1$, this is a local search algorithm.

\subsection{Heuristic search algorithm}

Clearly, the performance of the algorithm is expected to improve as $n^{*}$ increases, but the computational complexity makes $n^{*}>3$ not feasible for any practical problem with 1995 desktop computer technology, even if the local model structure set $\mathscr{L}$
contains as few as one or two possibilities.

Example. Consider the problem of identifying a state-space model of the form $x(t+1)=f(x(t))$, where $\operatorname{dim}(x)=5$, and we apply local models of the form

$$
\left(\begin{array}{c}
x_{1}(t+1) \\
x_{2}(t+1) \\
\vdots \\
x_{5}(t+1)
\end{array}\right)=\left(\begin{array}{c}
a_{1} \\
a_{2} \\
\vdots \\
a_{5}
\end{array}\right)+\left(\begin{array}{ccc}
a_{11} a_{12} & \ldots & a_{15} \\
a_{21} a_{22} & \ldots & a_{25} \\
\vdots & \ddots & \vdots \\
a_{51} a_{52} & \ldots & a_{55}
\end{array}\right)\left(\begin{array}{c}
x_{1}(t) \\
x_{2}(t) \\
\vdots \\
x_{5}(t)
\end{array}\right)
$$

Each of the 30 parameters can be replaced by a structural zero, which gives a set of local linear model structures with $2^{30} \approx 10^{9}$ elements. On the other hand, even if there 
is only one possible local model structure that one can choose, the number of possible decompositions into no more than five regimes is

$$
\# \mathscr{S}_{1}+\ldots+\# \mathscr{S}_{5}=1+d N_{1}+2\left(d N_{1}\right)^{2}+3 !\left(d N_{1}\right)^{2}+4 !\left(d N_{1}\right)^{4}
$$

For $d=2$ and $N_{1}=10$, this is approximately $4 \times 10^{6}$ candidate decompositions. With $n^{*}<4$, the model structure set is considerably reduced. In particular, $n^{*}=3$ gives about $10^{5}$ decompositions among which to search, $n^{*}=2$ gives about 2500 , while $n^{*}=1$ gives 80 candidate decompositions.

Because of the combinatorial nature of the model structure set, it is clearly of interest to implement some heuristics that cut down on the computational complexity without sacrificing too much of the optimality of the algorithm. As we have seen, the number of candidate decompositions at each step in the search may be large. To reduce the number of candidates, we suggest applying the following heuristics in the 'locally exhaustive' search at the second step in the search algorithm:

Heuristic 1. At each level in the search tree, proceed with only the most promising candidates.

The best candidates are of course not known a priori, so there is always a possibility that this may lead to a sub-optimal model. We suggest proceeding with the best decomposition for each of the possible splitting dimensions. Instead of trying to find the best candidates, one can often more easily single out the 'least promising candidate decompositions':

Heuristic 2. Discard the candidate decompositions that give an increase in the criterion from one level in the search tree to the next.

The number of remaining candidates will typically be larger than when using Heuristic 1, but the chance of discarding the optimal decomposition may be smaller. Some candidate decompositions may give rise to regimes where no substantial amount of data is available, and may therefore be classified a priori as not feasible:

Heuristic 3. Discard candidate decompositions that lead to regimes with few data points relevant to this regime compared to the number of degrees of freedom in the corresponding local model structure and local model validity function.

Counting the number of relevant data-points associated with each regime is controversial, since the interpolation functions overlap. We use the heuristic count $l_{i}=\Sigma_{t=1}^{l} w_{i}(z(t))$, which has the attractive property $\Sigma_{i \in I_{N}} l_{i}=l$.

Heuristic 4. Use a (backward or forward) stepwise regression procedure to handle local model structure sets $\mathscr{L}$ of combinatorial nature (Sugeno and Kang 1988).

Related to the example above, one should start with no structural zeroes, and then add one structural zero at a time, choosing the one that gives the largest improvement in the prediction performance. This should give less than $30+29+28+\ldots+1<30^{2}$ candidate model structures, which is quite different from $2^{30}$.

\subsection{User choices}

The basis-function $b(r ; \lambda)$ with scaling parameter $\lambda$ has the purpose of providing a smooth interpolation between the local models. The basis-function is assumed to have the property $b(r ; \lambda) \geqslant 0$ for all $r \in R$ and $b(r, \lambda) \rightarrow 0$ as $|r| \rightarrow \infty$. Typical choices are kernel-functions, like the unnormalized Gaussian $\exp \left(-r^{2} / 2 \lambda^{2}\right)$. It may appear that the choice of this function has significant impact on the model. However, it is our experience that the algorithm and model's prediction performance are quite insensitive 
with respect to this choice, and the specification of this function does not require any prior knowledge about the system. What is more important is the choice of $\lambda$, which is controlled by the user-specified parameter $\gamma$.

In order to compute the criterion $J_{G C V}$ or $J_{F P E}$, the effective number of parameters $p$ in the model structure must be known. If the choice of model structure is not based on the data, then the effective number of parameters is

$$
p=\sum_{i \in I_{N}} \operatorname{dim}\left(\theta_{i}\right)
$$

in the case of linear regression. However, the proposed algorithm for model structure identification makes use of the data $\mathscr{D}_{1}$ during the search. Hence, the $p$-value given by (13) will be too small. Counting the effective number of parameters in this case is controversial. We apply the heuristic

$$
p=\kappa(N-1)+\sum_{i \in I_{N}} \operatorname{dim}\left(\theta_{i}\right)
$$

where $\kappa \geqslant 0$ is a heuristic constant, which can be interpreted as a smoothing parameter, since a large $\kappa$ will put a large penalty on model complexity, and will therefore give a smooth model. A typical choice of $\kappa$ is between 0 and 4, see Friedman (1991).

\subsection{Statistical properties}

Consider the bias/variance decomposition (10). It has been shown in Johansen and Weyer (1995) that both the bias and variance will asymptotically (as $l \rightarrow \infty)$ tend towards their smallest possible values, with probability one, provided

1. The parameter estimator is consistent, see Ljung (1978) for conditions under which this holds.

2. The estimate $J^{\prime}$ of the expected squared prediction error used for model structure identification converges to its expectation.

3. Global minima of the parameter and structure optimization problems are found with probability one.

4. The model set $\left\{\mathscr{M}(\theta) ; \mathscr{M} \in \mathscr{S}, \theta \in \Theta_{\mathscr{M}}\right\}$ can be covered by a finite $\epsilon$-net.

It is known that the use of a separate validation data sequence for model structure identification gives a $J^{\prime}$ that satisfies the second requirement (Johansen and Weyer
1995).

Neither the parameter optimization not the structure optimization algorithms need result in global minima. An attractive feature of the model structure set is that it appears to have not only multiple global minima, but also many close-to-optimal local minima. It is easy to see that the restriction of the search to any sub-tree of the model structure tree does not exclude any possible decompositions into regimes. The worst thing that can happen is that the number of decompositions may be somewhat larger than necessary, or alternatively that the partition may not be as fine as desired. Obviously, this leads to suboptimality for finite amount of data, but not necessarily so asymptotically.

The fourth condition is somewhat technical, but it does in general impose a restriction on the complexity of the model set. In practice, this is not a serious restriction, as discussed in Johansen and Weyer (1995). 


\section{Examples}

\subsection{A simulated fermentation reactor}

Consider the fermentation of glucose to gluconic acid by the micro-organism Pseudomonas ovalis in a well stirred batch reactor. The main overall reaction mechanism is described by

$$
\begin{aligned}
& \text { Cells }+ \text { Glucose }+\mathrm{O}_{2} \rightarrow \text { More Cells } \\
& \text { Glucose }+\mathrm{O}_{2} \stackrel{\text { Cells }}{\rightarrow} \text { Gluconolactone } \\
& \text { Gluconolactone }+\mathrm{H}_{2} \mathrm{O} \rightarrow \text { Gluconic Acid }
\end{aligned}
$$

The production of gluconolactone is enzyme-catalysed by the cells. We use the following state-space model to simulate the 'true system' (Ghose and Ghosh 1976):

$$
\begin{aligned}
\dot{\chi} & =\mu_{m} \frac{s c}{k_{s} c+k_{0} s+s c} \chi \\
\dot{p} & =k_{p} l \\
\dot{l} & =v_{l} \frac{s}{k_{l}+s} \chi-0.91 k_{p} l \\
\dot{s} & =-\frac{1}{Y_{s}} \mu_{m} \frac{s c}{k_{s} c+k_{0} s+s c} \chi-1.011 v_{l} \frac{s}{k_{l}+s} \chi \\
\dot{c} & =k_{l} a\left(c^{*}-c\right)-\frac{1}{Y_{0}} \mu_{m} \frac{s c}{k_{s} c+k_{0} s+s c} \chi-0.09 v_{l} \frac{s}{k_{l}+s} \chi
\end{aligned}
$$

with initial conditions $\chi(0)=\chi_{0}, p(0)=0, l(0)=0, s(0)=s_{0}$ and $c(0)$ depending on the other initial conditions. The symbols and constants are defined in Table 1.

We simulated 10 hour batches using these equations, 'measuring' all states with 0.5 hour intervals, and adding sequentially uncorrelated random noise with a signal-tonoise ratio of approximately $30 \mathrm{~dB}$ to the states. We collected two sets of data, by

Table 1. Symbols and constants in the state-space simulation model for the fermenter.

\begin{tabular}{ll}
\hline Symbol & Description \\
\hline$\chi$ & Cell concentration [UOD/ml] \\
$p$ & Gluconic acid concentration $[\mathrm{g} / \mathrm{l}]$ \\
$l$ & Gluconolactone concentration $[\mathrm{g} / \mathrm{l}]$ \\
$s$ & Glucose concentration $[\mathrm{g} /]$ \\
$c$ & Dissolved oxygen concentration $[\mathrm{g} / \mathrm{l}]$ \\
$\mu_{m}$ & $0.39 \mathrm{~h}^{-1}$ \\
$k_{s}$ & $2.50 \mathrm{~g} / 1$ \\
$k_{0}$ & $0.55 \times 10^{-3} \mathrm{~g} / \mathrm{l}$ \\
$k_{p}$ & $0.645 \mathrm{~h}^{-1}$ \\
$v_{l}$ & $8.30 \mathrm{mg} \mathrm{UOD}^{-1} \mathrm{~h}^{-1}$ \\
$k_{l}$ & $12.80 \mathrm{~g} / 1$ \\
$k_{l} a$ & $150 \cdot 0-200 \cdot 0 \mathrm{~h}^{-1}$ \\
$Y_{s}$ & $0.375 \mathrm{UOD} / \mathrm{mg}$ \\
$Y_{o}$ & $0.890 \mathrm{UOD} / \mathrm{mg}$ \\
$c^{*}$ & $6.85 \times 10^{-3} \mathrm{~g} / 1$ \\
\hline
\end{tabular}


Table 2. Root average squared prediction performance for the model based on five operating regimes.

\begin{tabular}{lcc}
\hline State & One-step-ahead prediction & Ballistic prediction \\
\hline$\chi$ & 0.0134 & 0.0341 \\
$p$ & 0.0123 & 0.0211 \\
$l$ & 0.0157 & 0.0357 \\
$s$ & 0.0140 & 0.0204 \\
$c$ & 0.0137 & 0.0315 \\
\hline Total & 0.0139 & 0.0293 \\
\hline
\end{tabular}

randomly varying the initial conditions $\chi_{0}, s_{0}$ and the agitation speed (affecting $k_{l} a$ ). The first set contains data from 100 batches, and is used for system identification, while the second independent set is used for model testing. We define the following dimension-less normalized variables: $\chi=\chi /(3 \mathrm{UOD} / \mathrm{ml}), p_{n}=p /(50 \mathrm{~g} / \mathrm{l}), l_{n}=l /(15 \mathrm{~g} / \mathrm{l})$, $s_{n}=s /(50 \mathrm{~g} / \mathrm{l}), c_{n}=c /(0.01 \mathrm{~g} / \mathrm{l})$, and the normalized state-vector $x=\left(\chi_{n}, p_{n}, l_{n}, s_{n}, c_{n}\right)^{\mathrm{T}}$. We have specified only one possible local linear discrete-time state-space model structure $x(t+1)=a_{i}+A_{i} x(t)$ with 12 structural zeros in the $A_{i}$ matrices. These zeros follow directly from the reaction mechanism, see also Johansen and Foss (1993b). We observe that glucose and oxygen are rate-limiting and, consequently, expected to be the main contributors to the system's non-linearities. It follows that the operating point $z=\left(s_{n}, c_{n}\right)^{\mathrm{T}}$ captures these non-linearities and characterizes the operating conditions of the process with respect to local linear models, see also Johansen and Foss (1993b).

Running the identification algorithm with $n^{*}=1$, using the FPE criterion with $\kappa=1$, and Gaussian basis-function with $\gamma=1$, results in a model with five local models, and root average squared one-step-ahead prediction error (PE) on the test data $\mathrm{PE}=0.0139$, see Table 2. Restricting the number of local models to three, gives $\mathrm{PE}=0.0147$, while one global linear model gives $\mathrm{PE}=0.0303$. This clearly indicates that there exist significant non-linearities which have been captured by the two more complex models, and not by the linear model. The five regimes are illustrated in Fig. 3. Perhaps the most interesting and attractive feature of the method is that the

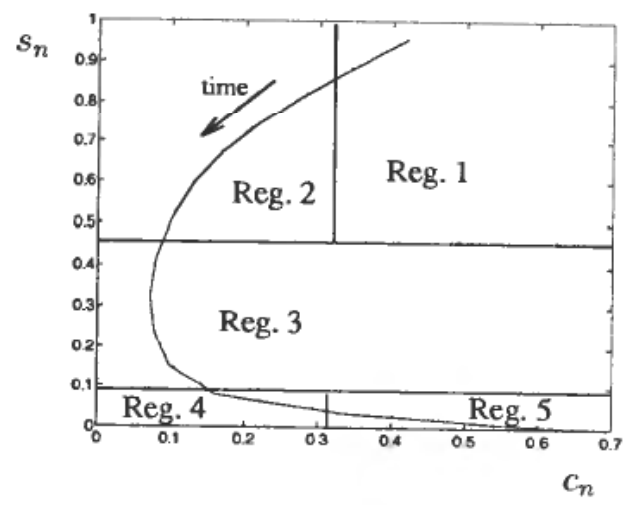

Figure 3. The decomposition into five regimes using the simulated fermenter data, with a typical simulated system trajectory projected onto the $\left(c_{n}, s_{n}\right)$-plane. 
identified model can be interpreted in a natural way. The five regimes correspond to the following phases in the batch

1. Initial phase.

2. Growth phase, where only the amount of micro-organisms is limiting the rate of the reactions.

3. Oxygen supply is rate-limiting.

4. Glucose is rate-limiting.

5. No glucose left, termination.

This gives a high-level qualitative description of the system. More low-level quantitative details on e.g. reaction kinetics can be added by examining the parameters of the local models corresponding to each regime. A simulation (ballistic prediction) of a typical batch from the test set is shown in Fig. 4, using the model with five local models, and the identified global linear model for comparison. Clearly, the results favour the non-linear model. Comparison with similar models with three or four regimes, designed by hand on the basis of qualitative system knowledge (Johansen and Foss 1993b) indicates that their performances are comparable. Although semi-empirical, the high transparency suggests that the identified model should not be viewed as a black-box.

The applied a priori knowledge is essentially the overall reaction mechanism, used for structuring the $A_{i}$ matrices and for selection of the two variables used for characterizing the operating space. It must be noted that the algorithm has also been applied without this knowledge, i.e. with full $A_{i}$ matrices and operating point $z=x$, resulting in only a slight decrease in the prediction accuracy of the identified model. In this case, it is interesting to observe that among the five states, the algorithm chooses only $\chi$ and $c$ to characterize the regimes. As noted in Johansen and Foss (1993b), due to the batch nature of the process operation, the information in the state $\chi$ is highly redundant, given the information in $s$. This is also evident from Fig. 4, which clearly shows the collinearity between these variables. Hence, the algorithm is forced to make a somewhat arbitrary choice about which variable to use for characterizing regimes, a fact that makes the interpretation of the model more difficult. This problem is also observed with the MARS algorithm (De Veaux et al. 1993). This only emphasizes the important fact that the success of empirical modeling is heavily dependent on the information in the empirical data, and that data deficiencies should to the highest possible extent be compensated for using prior knowledge.

\subsection{Modeling of a hydraulic manipulator}

A data sequence $\mathscr{D}_{8000}$ logged from a hydraulic TR4000 robot (Kavli 1990) from ABB Trallfa Robotics A/S was used to find a model describing the inverse dynamics

$$
\tau(t)=f(q(t) ; \dot{q}(t), \ddot{q}(t))+e(t)
$$

of a joint of this robot, where $\tau(t)$ is the control signal to the servo valve, $q(t)$ is joint position, and $e(t)$ is equation error. The joint position was logged at a sampling rate of $100 \mathrm{~Hz}$ while the robot was moving along a randomly generated trajectory. The joint velocity and acceleration was estimated by low-pass filtering and numerical differentiations. The prediction of an estimated linear model was subtracted from the data in order to emphasize the non-linearities. According to Kavli (1990), the non-linearities are mainly due to variations in the momentum arm of the hydraulic cylinder, non-linear damping, and non-linear pressure gain characteristics due to 


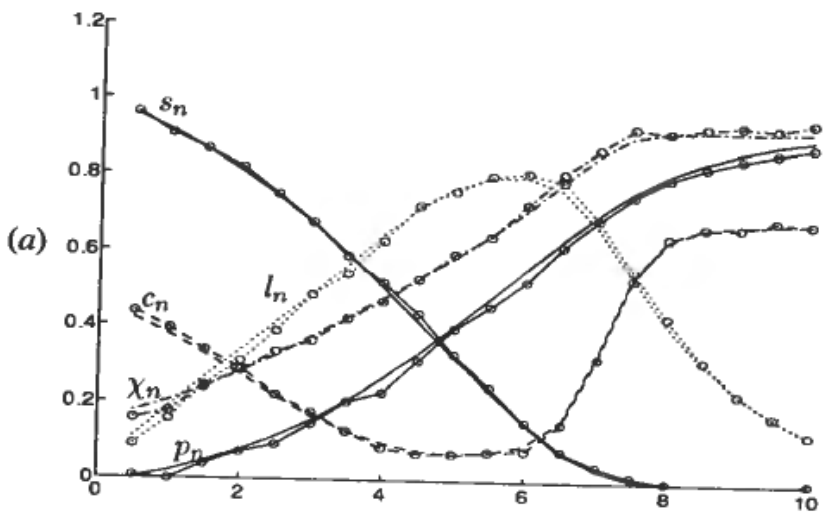

(b)
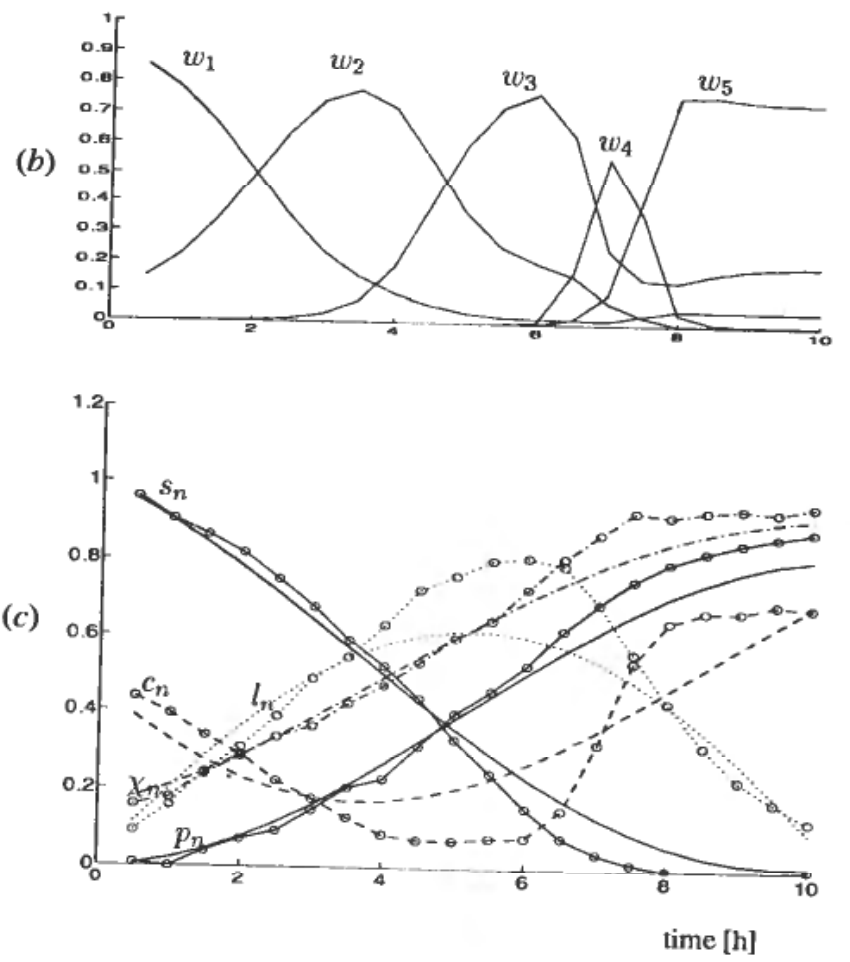

Figure 4. (a) Trajectories with circles are generated by the simulated 'true system', while the other trajectories are simulations of the model based on five local models. $(b)$ The relative weight of the various local models in the interpolation. (c) Simulation of the
identified global linear model. 
Table 3. Results of applying various empirical modeling algorithms on the hydraulic manipulator joint data. The NRMSE criterion is defined as the square root of the ratio of the average squared one-step-ahead prediction error to the variance of the output, using the independent test data.

\begin{tabular}{llcc}
\hline Model & Comments & Num. param. & NRMSE \\
\hline ASMOD & Quadratic Spline Basis & 561 & $15 \%$ \\
MARS & & 155 & $16 \%$ \\
Local linear & $\gamma=1, n^{*}=2$, Heuristics 1, 2, and 3 & 80 & $17 \%$ \\
Local linear & $\gamma=1, n^{*}=1$, Heuristic 3 & 80 & $17 \%$ \\
MARS & & 53 & $17 \%$ \\
Local linear & $\gamma=1, n^{*}=2$, Heuristics 1, 2 and 3 & 40 & $18 \%$ \\
Local linear & $\gamma=1, n^{*}=1$, Heuristic 3 & 40 & $19 \%$ \\
RBF $^{+}$ & Gaussian radial basis-functions & 112 & $19 \%$ \\
MARS & & 16 & $20 \%$ \\
ASMOD & & 48 & $20 \%$ \\
Local linear & $\gamma=1, n^{*}=2$, Heuristics 1, 2 and 3 & 20 & $23 \%$ \\
NN $^{+}$ & Sigmoidal Neural Network (3-20-1) & 101 & $23 \%$ \\
Local linear $^{+}$ & $\gamma=1, n^{*}=1$, Heuristic 3 & 20 & $26 \%$ \\
NN $^{+}$ & Sigmoidal Neural Network (3-5-1) & 26 & $26 \%$ \\
\hline
\end{tabular}

varying flow-rates in the servo valve. In addition, 1000 independent samples were used for testing the model. A number of models were identified, based on the structure model structure, and a Gaussian basis-function with $\gamma=1$. The results are summarized and compared to the results with the ASMOD algorithm in Kavli (1993) (marked $^{\ddagger}$ ), Carlin et al. (1994) (marked $^{+}$), and the MARS algorithm, Friedman (1991) in Table 3. The table shows that the structure identification algorithm is able to find an adequate model with a small number of parameters while maintaining the high accuracy of the models found by MARS and ASMOD. In all cases, only the parameters corresponding to local model parameters or basis-function coefficients are counted in the table. Notice that according to the FPE criterion with $\kappa=1$, the data sequence allows more degrees of freedom to be added to the identified model structures. This was not pursued due to the computational complexity involved. The operating point was chosen as $z=(q$, $\dot{q}, \ddot{q})$ although complementary identification experiments showed that $z=(\dot{q}, \ddot{q})$ was sufficient to capture most of the non-linearities, in particular when using no more then 40 parameters. Unfortunately, the large number of regimes makes the interpretation of the empirical model more difficult than in the previous example. The model should be viewed as a black box. This example mainly serves as a benchmark that shows that the accuracy achieved with the local modeling approach is comparable to some of the most popular empirical modeling algorithms from the literature.

\section{Discussion}

The amount of prior knowledge required with the proposed approach is quite reasonable. First of all, an operating point space $Z$ is required. In many cases, it is possible to choose $Z$ equal to a subspace or sub-manifold of the input space (Johansen and Foss 1993a). The design of $Z$ need not be based solely on a priori knowledge, but can in addition consider the distribution of the data $\mathscr{D}_{1}$. Quite often, there are collinearities or correlations in the data, so that $\mathscr{D}_{I}$ can be embedded in a subspace or sub-manifold of considerably lower dimension than the input space. In that case, $z$ need not be of higher dimension than this embedding. Some prior knowledge will often make 
it possible to reduce the dimension of $z$ considerably. This is important, since it may both reduce the complexity of the model and improve its transparency, and also reduce the computational complexity for the empirical modeling algorithm considerably.

A set of local model structure candidates must be specified. If no a priori knowledge exists to support one choice over the other, one will typically choose local linear model structures of various orders and possibly with structural zeros as default, since linear models are well understood and possible to interpret. Moreover, a linear model will always be a sufficiently good approximation locally, provided the system is smooth, and the regimes are small enough. On the other hand, if there is substantial a priori knowledge available in terms of mechanistic local model structures, these can be included as illustrated in Johansen (1994). Such local model structures may for example be simplified mass- and energy-balances.

The purposes of a model can be diverse, e.g. system analysis, design, optimization, prediction, control, or diagnosis. In many applications, it is important that the model can be easily interpreted and understood in terms of the system mechanisms. With empirical models, which are often based on black-box model representations, this is often a hard or impossible task. However, the approach presented in this paper gives highly transparent empirical models because

- local models are simple enough to be interpreted,

- the operating regimes constitute a qualitative high-level description of the system that is close to engineering thinking.

Notice that the interpretation of local linear models as linearizations of the system at various operating points is valid only if the model validity functions do not overlap too much. This point is stressed in Murray-Smith (1994), and the use of local identification algorithms for each local model is one possibility that will improve the interpretability.

\subsection{Related work}

Linear models are applied in Jones et al. (1991) and Stokbro et al. (1990) together with a clustering algorithm to determine the location of the local models. A parametrized regime description and a hierarchical estimator used to estimate the regime parameters simultaneously with the local model parameters is described in Jacobs et al. (1991) and Jordan and Jacobs (1993). An algorithm based on local linear models and decomposition of regimes in which the system appears to be more complex than the model, is suggested in Murray-Smith and Gollee (1994). A model representation based on local polynomial models and smooth interpolation is proposed in Pottmann et al. (1993). The structure identification algorithm is based on an orthogonal regression algorithm that sequentially discards local model terms that are found to have small significance.

When the interpolation functions are chosen as the characteristic functions of the regime-sets $Z_{i}$, a piecewise linear model results. The resulting model will not be smooth, and may not even be continuous, which may be a requirement in some applications. Also, we have experienced that smooth interpolation between local linear models usually gives better model fit compared to a piecewise linear model with the same number of parameters. The local linear modeling approach combined with a fuzzy set representation of the regimes also leads to a model representation with interpolation between the local linear models (Takagi and Sugeno 1985). In that case, it is the fuzzy 
inference mechanism that implicitly gives an interpolation. Structure identification algorithms based on clustering (Bezdek et al. 1981, Yoshinari 1993, Yager and Filev 1993) and local search (Sugeno and Kang 1988) have been proposed in this context. In this case, the $\rho_{i}$-functions are interpreted as membership functions for fuzzy sets.

The algorithm of Sugeno and Kang (1988) is perhaps the closest relative to the present algorithm. The main difference is the extra flexibility, and effort is being applied to find a closer to optimal model with the present algorithm. A statistical pattern recognition approach with multiple models leads to a similar representation based on a piecewise linear model and discriminant functions to represent the regime boundaries (Skeppstedt et al. 1992). Finally, in Sorheim (1990) it is suggested a model representation with neural nets as local models and a structure identification algorithm based on pattern recognition. The pattern recognition algorithm will detect parts of the input space in which the model fit is inadequate, and refine the model locally.

With this large body of literature in mind, one may ask: What are the contributions and improvements represented by the present approach? We have attempted to take the most attractive features from the algorithms in the literature and combined these into one algorithm. We have emphasized interpretability of the resulting model, flexibility with respect to incorporation of prior knowledge, and a transparent modeling and identification process that is close to engineering thinking. The price we have to pay is a computer intensive algorithm. Some may also argue that the algorithm is too flexible and not completely automatic, and as a result it may be difficult to apply for inexperienced users. However, it is our view that real world applications require perhaps even more flexibility and a less automated approach.

\section{Concluding remarks}

The proposed empirical modeling and identification algorithm is based on a rich non-linear model representation which utilizes local models and interpolation to represent a global model. With this representation, the semi-empirical modeling problem is solved using a structure identification algorithm based on a heuristic search for decompositions of the system's operating range into operating regimes. This algorithm is the main contribution of this work. We want to emphasize an important property of the modeling method and identification algorithm, namely the transparency of the resulting model. The transparency is linked with the possibility to interpret each of the simple local models independently, but more importantly with the fact that the identified regimes can often be interpreted in terms of the system behaviour or mechanisms.

In general, the fundamental assumptions behind empirical modeling are that (1) the empirical data is not too contaminated by noise and other unmodeled phenomena, and (2) that the data set is complete in the sense that it contains a sufficient amount of information from all interesting operating conditions and system variables. Unfortunately, these assumptions are often not met in practical applications. The proposed algorithm should therefore be applied with care, and as a part of a computer aided modeling environment (Johansen 1995) that allows flexible incorporation of prior knowledge, not as an automatic modeling algorithm. Moreover, one should undertake a study of the robustness of the algorithm with respect to contaminated, sparse, and incomplete data, in particular for high dimensional and otherwise complex modeling problems. 


\section{ACKNOWLEDGMENTS}

This work was partly supported by The Research Council of Norway under doctoral scholarship grant no. ST. 10.12.221718 given to the first author. We want to thank Dr. Tom Kavli and several of his colleagues at SINTEF-SI for valuable discussions and making the hydraulic manipulator data available.

\section{REFERENCES} AKAIKE, H. (1969). Fitting autoregressive models for prediction. Ann. Inst. Stat. Math, 21,
243-247.

BEZDEK, J.C., CORAY, C., GundERSON, R., and WATSON, J. (1981). Detection and characterization of cluster substructure. II. Fuzzy c-varieties and complex combinations thereof. SIAM J. Applied Mathematics, 40, 352-372.

Carlin, M., KaVli, T., and Lillekjendlie, B. (1994). A comparison of four methods for non-linear data modeling. Chemometrics and Int. Lab. Sys., 23, 163-178.

Craven, P., and WAHBA, G. (1979). Smoothing noisy data with spline functions. Estimating the correct degree of smoothing noisy data with spline functions. Estimating the correct degree of smoothing by the method of generalized cross-validation. Numerical Math., 31,

De Veaux, R. D., Psichogios, D. C., and Ungar, L. H. (1993). A tale of two nonparametric estimation schemes: MARS and neural networks. In Proc. 4th Int. Conf. Artificial Intelligence and Statistics.

FriEdMAN, J. H. (1991). Multivariable adaptive regression splines (with discussion). The Annals of Statistics, 19, 1-141.

Ghose, T. K., and GHosh, P. (1976). Kinetic analysis of gluconic acid production by pseudomonas ovalis. J. Applied Chemical Biotechnology, 26, 768-777.

JACOBS, R. A., JoRDAN, M. I., Nowlan, S. J., and HiNTON, G. E. (1991). Adaptive mixtures of local experts. Neural Computation, 3, 79-87.

JOHANSEN, T. A., and Foss, B. A. (1993a). Constructing NARMAX models using ARMAX models. Int. J. Control, 58, 1125-1153.

JOHANSEN, T. A., and Foss, B. A. (1993b). State-space modeling using operating regime decomposition and local models. In Preprints 12th IFAC World Congress, Sydney, Australia, 1, 431-434.

JohAnsen, T. A. (1994). Operating Regime Based Process Modeling and Identification. Dr. Ing Thesis, Department of Engineering Cybernetics, Norwegian Institute of Technology. Available on http://www.itk.unit.no/ansatte/Johansen,Tor.Arne/dring-taj.ps.gz.

JOHANSEN, T. A. (1995) Operating Regime Based Modeling and Identification Toolbox-User's Guide and Reference. Technical Report STF, SWTEF, Trondheim.

JOHANSEN, T. A., and WEYER, E. (1995). Model structure identification using separate validation data-asymptotic properties. Proceedings of the European Control Confer-
ence, Rome.

JONES, R. D., et al. (1991). Nonlinear adaptive networks: A little theory, a few applications. Technical Report 91-273, Los Alamos National Lab., NM, USA.

JORDAN, M. I., and JACOBS, R. A. (1993). Hierarchical mixtures of experts and the EM algorithm. technical Report 9301, MIT Computational Cognitive Science, Cambridge, MA, USA.

KAVLI, T. (1990). Nonuniformly partitioned piecewise linear representation of continuous learned mappings. In Proceedings of IEEE Int. Workshop on Intelligent Motion Control,
Istanbul, pp. 115-122.

KAVLI, T. (1993). ASMOD-An algorithm for adaptive spline modeling of observation data. Int. J. Control, 58, 947-967.

LARSEN, J. (1992). A generalization error estimate for nonlinear systems. In Proc. IEEE Workshop on Neural Networks for Signal Processing, Piscataway, NJ, pp. 29-38.

LJUNG, L. (1978). Convergence analysis of parametric identification methods. IEEE Trans. Automatic Control, 23, 770-783. LJUNG, L. (1987). System Identification: Theory for the User. (Prentice-hall, Inc., Englewood
Cliffs, NJ). MurRaY-SMith, R., (1994). Local model networks and local learning. In Proceedings of Fuzzy
Duisburg, pp. 404409. 
Murray-Smith, R., and GolleE, H. (1994). A constructive learning algorithm for local model networks. In Proceedings of the IEEE Workshop on Computer-Intensive Methods in Control and Signal Processing, Prague, Czech Republic, pp. 21-29.

Pottmann, M. Unbehauen, H., and Seborg, D. E. (1993). Application of a general multi-model approach for identification of highly non-linear processes-A case study. Int. J. Control, 57, 97-120.

Skeppstedt, A., LuUnG, L., and MillnERT, M. (1992). Construction of composite models from observed data. Int. J. Control, 55, 141-152.

SøRHEIM, E. (1990). A combined network architecture using ART2 and back propagation for adaptive estimation of dynamical processes. Modeling, Identification and Control, 11, 191-199.

Stoica, P., EykHoff, P., JANSSEN, P., and SÖDERSTRÖM, T. (1986). Model-structure selection by cross-validation. Int. J. Control, 43, 1841-1878.

STOKBro, K., HerTz, J. A., and UMBERGER, D. K. (1990). Exploiting neurons with localized receptive fields to learn chaos. J. Complex Systems., 4, 603.

STONE, M. (1974). Cross-validatory choice and assessment of statistical predictions. J. Royal Statistical Soc. B, 36, 111-133.

SugENO, M., and KANG, G. T. (1988). Structure identification of fuzzy model. Fuzzy Sets and Systems, 26, 15-33.

TAKAGI, T., and SugENo, M. (1985). Fuzzy identification of systems and its application to modeling and control. IEEE Trans. Systems, Man, and Cybernetics., 15, 116-132.

YAGER, R. R., and FILEV, D. P. (1993). Unified structure and parameter identification of fuzzy models. IEEE Trans. Systems, Man, and Cybernetics, 23, 1198-1205.

Yoshinar1, Y., PEDRYCZ, W., and Hirota, K. (1993). Construction of fuzzy models through clustering techniques. Fuzzy Sets and Systems, 54, 157-165. 